\title{
HARDY INTEGRAL ESTIMATES FOR THE LAPLACE TRANSFORM
}

\author{
STEVEN BLOOM
}

(Communicated by Andrew M. Buckner)

\begin{abstract}
Weighted norm inequalities for the Laplace transform are obtained by reducing the Laplace operator to the Hardy operator. The conditions obtained include necessary and sufficient conditions when one of the weights is doubling, including, in particular, all the power weights.
\end{abstract}

In this note, we would like to describe some simple methods for reducing $L^{p}$ estimates for the Laplace transform to the much simpler Hardy antidifferentiation operator. This yields fairly sharp weighted norm inequalities for the Laplace transform.

Let $\mathscr{L} f(x)=\int_{0}^{\infty} e^{-x t} f(t) d t$ be the Laplace transform of $f$, for $0<x<\infty$. The Hardy operator $H f$ is given by $H f(x)=\int_{0}^{x} f(t) d t$. Let $H^{*}$ denote the adjoint operator of $H, H^{*} f(x)=\int_{x}^{\infty} f(t) d t$. By a weight, we mean a nonnegative function. The main results of this note are the following two theorems.

Theorem 1. Let $v$ and $w$ be weights defined on $(0, \infty)$. In order for the Laplace transform $\mathscr{L}: L^{p}(v) \rightarrow L^{q}(w)$ boundedly, set $u(x)=x^{-2} w(1 / x)$.

(a) If $1<p \leq q<\infty$, it is necessary that

$$
H\left(v^{-p^{\prime} / p}\right)^{1 / p^{\prime}} H^{*}(u)^{1 / q} \leq C
$$

and sufficient that

$$
H\left[\left(v^{-1}\right)^{* p^{\prime} / p}\right]^{1 / p^{\prime}} H^{*}(u)^{1 / q} \leq C,
$$

where the ${ }^{*}$ on the weight denotes the decreasing rearrangement of $v^{-1}$. The prime denotes the conjugate exponent, $1 / p+1 / p^{\prime}=1$.

(b) If $1<q<p<\infty$ and if $1 / r=1 / q-1 / p$, it is necessary that

$$
\int_{0}^{\infty} v^{-p^{\prime} / p}\left[H\left(v^{-p^{\prime} / p}\right)^{1 / q^{\prime}} H^{*}(u)^{1 / q}\right]^{r}<\infty
$$

and sufficient that

$$
\int_{0}^{\infty}\left(v^{-1}\right)^{* p^{\prime} / p}\left[H\left(\left(v^{-1}\right)^{* p^{\prime} / p}\right)^{1 / q^{\prime}} H^{*}(u)^{1 / q}\right]^{r}<\infty .
$$

Received by the editors April 9, 1990 and, in revised form, March 6, 1991.

1991 Mathematics Subject Classification. Primary 26D15, 26 A33.

Research supported in part by a grant from Siena College. 
Theorem 2. In order for $\mathscr{L}: L^{p}(v) \rightarrow L^{q}(w)$ boundedly,

(a) If $1<p \leq q<\infty$, it is necessary that

$$
H\left(v^{-p^{\prime} / p}\right)(x)^{1 / p^{\prime}} \mathscr{L} w(q x)^{1 / q} \leq C
$$

and sufficient that

$$
H\left(v^{-p^{\prime} / p}\right)(x)^{1 / p^{\prime}} \mathscr{L} w(x)^{1 / q} \leq C .
$$

(b) If $1<q<p<\infty$ and if $1 / r=1 / q-1 / p$, it is necessary that

$$
\int_{0}^{\infty} v^{-p^{\prime} / p}\left[H\left(v^{-p^{\prime} / p}\right)^{1 / q^{\prime}} \mathscr{L} w(q x)^{1 / q}\right]^{r} d x<\infty
$$

and sufficient that

$$
\int_{0}^{\infty} v^{-p^{\prime} / p}\left[H\left(v^{-p^{\prime} / p}\right)^{1 / q^{\prime}} \mathscr{L} w(x)^{1 / q}\right]^{r} d x<\infty .
$$

Some remarks. (1.9) The conditions (1.1)-(1.8) are simply the corresponding Hardy conditions for various weights. Both theorems are essentially reductions of the $L^{p}$-mapping properties of the Laplace transform to the much simpler Hardy operator.

(1.10) Since the Laplace transform is self-adjoint, there are dual versions to both theorems. Thus Theorem 1 gives necessary and sufficient conditions when the domain weight $v$ is increasing, and its dual version can be used when the range weight $w$ is decreasing.

(1.11) Theorem 1 is quite elementary, and although it appears to be new, its proof is more or less implicit in papers like [1] or [5]. We have included it because it nicely illustrates the idea of reducing the Laplace transform to the Hardy operator.

(1.12) Theorem 2 gives necessary and sufficient conditions whenever the range weight $w$ is such that $\mathscr{L} w(2 x) \approx \mathscr{L} w(x)$. A simple change of variables shows that this holds whenever $w$ satisfies the doubling condition

$$
w(2 x) \leq C w(x)
$$

which includes all decreasing $w$ 's as well as the increasing power weights. So, in view of remark (1.10), Theorem 2 supercedes Theorem 1. It is, in essence, a stronger result than Theorem 1 . Needless to say, its proof is much more difficult.

In fact, this equivalence holds whenever $w$ is doubling in the more traditional sense. Call the weight $w$ doubling at zero if

$$
\int_{0}^{2 x} w(t) d t \leq C \int_{0}^{x} w(t) d t
$$

for every $x>0$. For such weights,

$$
\mathscr{L} w(x) \leq C \mathscr{L} w(2 x)
$$

for every $x>0$. Here is a proof:

(1.13) is vacuous, but true, if $\mathscr{L} w \equiv \infty$. So we may postulate some $x_{0}>$ 0 with $\mathscr{L} w\left(x_{0}\right)$ finite. It will be sufficient to prove (1.13) for any $x$ with $\mathscr{L} w(2 x)<\infty$. Fix such an $x$. Then

$$
\mathscr{L} w(x)=2 \int_{0}^{\infty} e^{-2 x t} w(2 t) d t
$$


(we will worry about the convergence of this integral later). Choose $\delta$ so that $e^{x \delta}=2$, and set

$$
I_{n}=[(n-1) \delta, n \delta)
$$

So

$$
\mathscr{L} w(x) \leq 4 \sum_{n=1}^{\infty} 2^{-n} \int_{I_{n}} w(2 t) d t
$$

and (1.13) will follow, including the existence of $\mathscr{L} w(x)$, if we can bound the partial sums $S_{N}$ of this series by a constant times $\mathscr{L} w(2 x)$. But

$$
\begin{aligned}
S_{N} & =2^{-N} \sum_{n=1}^{N} 2^{(N-n)} \int_{I_{n}} w(2 t) d t=2^{-N} \sum_{k=0}^{N-1} 2^{k} \int_{I_{N-k}} w(2 t) d t \\
& \leq 2^{-N} \sum_{k=0}^{N-1} \sum_{j=0}^{k} 2^{j} \int_{I_{N-k}} w(2 t) d t=2^{-N} \sum_{j=0}^{N-1} \sum_{k=j}^{N-1} 2^{j} \int_{I_{N-k}} w(2 t) d t \\
& =2^{-N} \sum_{j=0}^{N-1} 2^{j} \int_{0}^{(N-j) \delta} w(2 t) d t \\
& \leq C 2^{-N} \sum_{j=0}^{N-1} 2^{j} \int_{0}^{(N-j) \delta} w(t) d t \quad \text { by the doubling at zero } \\
& =C 2^{-N} \sum_{k=0}^{N-1} \sum_{j=0}^{k} 2^{j} \int_{I_{N-K}} w(t) d t \leq 2 C 2^{-N} \sum_{k=0}^{N-1} 2^{k} \int_{I_{N-k}} w(t) d t \\
& =2 C \sum_{n=1}^{N} 2^{-n} \int_{I_{n}} w(t) d t \leq 2 C \sum_{n=1}^{N} \int_{I_{n}} e^{-2 x t} w(t) d t \leq 2 C \mathscr{L} w(2 x) .
\end{aligned}
$$

We give the proofs in the next section and describe some power weight examples in $\S 3$.

\section{Proofs}

We will, of course, need the weighted norm inequality conditions for the Hardy operator, contained in [2, 4, 6-9]. These are:

Theorem 2.1. $H: L^{p}(v) \rightarrow L^{q}(u)$ boundedly if and only if

(a) when $1<p \leq q<\infty$,

$$
H\left(v^{-p^{\prime} / p}\right)^{1 / p^{\prime}} H^{*}(u)^{1 / q} \leq C,
$$

or

(b) when $1<q<p<\infty$ and $1 / r=1 / q-1 / p$,

$$
\int_{0}^{\infty} v^{-p^{\prime} / p}\left[H\left(v^{-p^{\prime} / p}\right)^{1 / q^{\prime}} H^{*}(u)^{1 / q}\right]^{r} d x<\infty .
$$

Proof of Theorem 1. Comparing Theorems 1 and 2.1, it is clear that we must show that

$$
H: L^{p}\left(\frac{1}{v^{-1 *}}\right) \rightarrow L^{q}(u) \quad \text { is sufficient }
$$


with

$$
H: L^{p}(v) \rightarrow L^{q}(u) \quad \text { necessary . }
$$

Suppose (2.2) holds. Since the kernel $e^{-x t}$ is decreasing,

$$
|\mathscr{L} f(x)| \leq \mathscr{L}(|f|)(x) \leq \mathscr{L} f^{*}(x) .
$$

Also

$$
\int\left(f^{*}\right)^{p}\left(v^{-1 *}\right)^{-1} \leq \int|f|^{p} v .
$$

So it suffices to show that

$$
\left(\int\left(\mathscr{L} f^{*}\right)^{q} w\right)^{1 / q} \leq C\left(\int\left(f^{*}\right)^{p}\left(v^{-1 *}\right)\right)^{1 / p}
$$

But,

$$
\begin{aligned}
\mathscr{L} f^{*}(x) & \leq H f^{*}\left(\frac{1}{x}\right)+\int_{1 / x}^{\infty} e^{-x t} f^{*}(t) d t \leq H f^{*}\left(\frac{1}{x}\right)+f^{*}\left(\frac{1}{x}\right) \int_{1 / x}^{\infty} e^{-x t} d t \\
& =H f^{*}\left(\frac{1}{x}\right)+\frac{1}{e \cdot x} f^{*}\left(\frac{1}{x}\right) \leq\left(1+e^{-1}\right) H f^{*}\left(\frac{1}{x}\right)
\end{aligned}
$$

and so,

$$
\|\mathscr{L} f\|_{L^{q}(w)} \leq\left(1+e^{-1}\right)\left[\int_{0}^{\infty} H f^{*}\left(\frac{1}{x}\right)^{q} w(x) d x\right]^{1 / q}=\left(1+e^{-1}\right)\left\|H f^{*}\right\|_{L^{q}(u)}
$$

and (2.4) follows from (2.2).

Conversely, for $f \geq 0, \mathscr{L} f(x) \geq \int_{0}^{1 / x} e^{-x t} f(t) d t \geq \frac{1}{e} H f\left(\frac{1}{x}\right)$ and (2.3) must hold after the obvious change of variable.

As promised, this was quite simple. Theorem 2 will be derived from a series of lemmas, using techniques similar to those in [3].

Lemma 2.5. Let $f \geq 0$ and let $w$ be a weight. Then

Proof. Taking adjoints,

$$
\int_{0}^{\infty}(\mathscr{L} f)^{2} w=2 \int_{0}^{\infty} f(x) \int_{0}^{x} f(t) \mathscr{L} w(x+t) d t d x
$$

$$
\int(\mathscr{L} f)^{2} w=\int f \mathscr{L}(w \mathscr{L} f) .
$$

Now,

$$
\begin{aligned}
\mathscr{L}(w \mathscr{L} f)(x) & =\int_{0}^{\infty} e^{-x t} w(t) \int_{0}^{\infty} e^{-s t} f(s) d s d t \\
& =\int_{0}^{\infty} f(s) \int_{0}^{\infty} e^{-(x+s) t} w(t) d t=\int_{0}^{\infty} f(s) \mathscr{L} w(x+s) d s
\end{aligned}
$$

and so

$$
\begin{aligned}
\int(\mathscr{L} f)^{2} w & =\int_{0}^{\infty} f(x)\left(\int_{0}^{x}+\int_{x}^{\infty}\right) f(t) \mathscr{L} w(x+t) d t d x \\
& =2 \int_{0}^{\infty} f(x) \int_{0}^{x} f(t) \mathscr{L} w(x+t) d t d x
\end{aligned}
$$


by another Fubini, and the proof is complete.

Lemma 2.6. Let $f \geq 0$, let $w$ be a weight, and let $p \geq 2$. Then

$$
\int_{0}^{\infty}(\mathscr{L} f)^{p} w \leq 2^{p-1} \int_{0}^{\infty} f(x) H f(x)^{p-1} \mathscr{L} w(x) d x .
$$

Proof. This follows trivially from Lemma 2.5 when $p=2$ since $\mathscr{L} w(x+t) \leq$ $\mathscr{L} w(x)$. So consider $p>2$. We will assume at first that $\int(\mathscr{L} f)^{p} w<\infty$.

Let $u(x)=w(x)(\mathscr{L} f)(x)^{p-2}$. By the simple $p=2$ case,

$$
\int(\mathscr{L} f)^{p} w \leq 2 \int f H f \mathscr{L} u .
$$

We intend to apply Hölder's inequality twice with exponents $(p-1) /(p-2)$ and $p-1$.

$$
\begin{aligned}
\mathscr{L} u(x) & =\int_{0}^{\infty} e^{-x t}(\mathscr{L} f)(t)^{p-2} w(t) d t \\
& \leq(\mathscr{L} w)(x)^{1 /(p-1)}\left[\mathscr{L}\left[w(\mathscr{L} f)^{p-1}\right](x)\right]^{(p-2) /(p-1)},
\end{aligned}
$$

and so

$$
\begin{aligned}
\int(\mathscr{L} f)^{p} w & \leq 2 \int f H f(\mathscr{L} w)^{1 /(p-1)}\left(\mathscr{L}\left[w(\mathscr{L} f)^{p-1}\right]\right)^{(p-2) /(p-1)} \\
& \leq 2\left[\int f(H f)^{p-1} \mathscr{L} w\right]^{1 /(p-1)}\left(\int f \mathscr{L}\left[w(\mathscr{L} f)^{p-1}\right]\right)^{1-1 /(p-1)} \\
& =2\left[\int f(H f)^{p-1} \mathscr{L} w\right]^{1 /(p-1)}\left[\int(\mathscr{L} f)^{p} w\right]^{1-1 /(p-1)},
\end{aligned}
$$

and since $\int(\mathscr{L} f)^{p} w<\infty$, this gives

$$
\left[\int(\mathscr{L} f)^{p} w\right]^{1 /(p-1)} \leq 2\left[\int f(H f)^{p-1} \mathscr{L} w\right]^{1 /(p-1)}
$$

as the lemma asserts.

So the proof will be complete if we can show that whenever $\int f(H f)^{p-1} \mathscr{L} w$ $<\infty$, so is $\int(\mathscr{L} f)^{p} w<\infty$.

To this end, fix such an $f$, which we can assume with no loss of generality to be continuous since if the lemma holds for all continuous $f$ it holds for all $f$. Let $a$ be the smallest number for which $f>0$ in some neighborhood $(a, a+\varepsilon)$. Hence $f$ vanishes in $(0, a]$, and so

$$
\int f(H f)^{p-1} \mathscr{L} w=\int_{a}^{\infty} f(x) H f(x)^{p-1} \mathscr{L} w(x) d x<\infty .
$$

This forces $\mathscr{L} w(x)<\infty$ a.e. for $a<x<a+\varepsilon$, and since $\mathscr{L} w(x)$ decreases, it must be finite for all $x>a$.

We can approximate $f$ by $g$ continuous and compactly supported in $(a, \infty)$. It will suffice to show that $\int(\mathscr{L} g)^{p} w<\infty$ for all such $g$, for then we have shown that

$$
\int(\mathscr{L} g)^{p} w \leq 2^{p-1} \int g(H g)^{p-1} \mathscr{L} w,
$$


and we get this for $f$. So fix a $g$ supported in $(b, c)$ and $b>a$. By the $p=2$ case, which used no finiteness assumptions,

$$
\int(\mathscr{L} g)^{p} w \leq 2 \int_{b}^{\infty} g H g \mathscr{L}\left[w(\mathscr{L} g)^{p-2}\right] .
$$

Now each $\mathscr{L} g(t) \leq\|g\|_{1}$, and so for $x \geq b$,

$$
\mathscr{L}\left[w(\mathscr{L} g)^{p-2}\right](x) \leq\|g\|_{1}^{p-2} \mathscr{L} w(x) \leq\|g\|_{1}^{p-2} \mathscr{L} w(b),
$$

and so

$$
\int_{b}^{\infty} g H g \mathscr{L}\left[w(\mathscr{L} g)^{p-2}\right] \leq\|g\|_{1}^{p} \mathscr{L} w(b)<\infty,
$$

proving the lemma.

Lemma 2.7. Let $f \geq 0$, let $w$ be a weight, and let $1<p<2$. Then

$$
\int(\mathscr{L} f)^{p} w \leq \frac{2}{p-1} \int f(H f)^{p-1} \mathscr{L} w
$$

Proof. By the proof of Lemma 2.5,

$$
\int(\mathscr{L} f)^{p} w=2 \int_{0}^{\infty} f(x) \int_{0}^{x} f(t) \mathscr{L}\left[w(\mathscr{L} f)^{p-2}\right](x+t) d t d x .
$$

Now,

$$
\begin{aligned}
\mathscr{L}\left[w(\mathscr{L} f)^{p-2}\right](x+t) & =\int_{0}^{\infty} e^{-(x+t) s} w(s)\left(\int_{0}^{\infty} e^{-y s} f(y) d y\right)^{p-2} \\
& \leq \int_{0}^{\infty} e^{-(x+t) s} w(s)\left(\int_{0}^{t} e^{-s t} f(y) d y\right)^{p-2}, \quad \text { since } p<2, \\
& =H f(t)^{p-2} \int_{0}^{\infty} e^{-x s} e^{-(p-1) t s} w(s) d s \\
& \leq H f(t)^{p-2} \mathscr{L} w(x) .
\end{aligned}
$$

Hence,

$$
\int(\mathscr{L} f)^{p} w \leq 2 \int_{0}^{\infty} f(x) \mathscr{L} w(x) \int_{0}^{x} f(t) H f(t)^{p-2} d t d x
$$

But,

$$
f(t) H f(t)^{p-2}=\frac{d}{d t} \frac{1}{p-1} H f(t)^{p-1},
$$

and integrating this out gives the lemma.

Lemma 2.8. Let $f \geq 0$, let $w$ be a weight, and let $p \geq 2$. Then

$$
\int(\mathscr{L} f)^{p} w \geq 2 \int f(H f)(x)^{p-1} \mathscr{L} w(p x) d x
$$


Proof. The case $p=2$ again follows easily from Lemma 2.5, since here $\mathscr{L} w(x+t) \geq \mathscr{L} w(2 x)$. Applying this when $p>2$ gives

$$
\begin{aligned}
\int(\mathscr{L} f)^{p} w & \geq 2 \int f H f(x) \mathscr{L}\left[w(\mathscr{L} f)^{p-2}\right](2 x) d x \\
& =2 \int_{0}^{\infty} f(x) H f(x) \int_{0}^{\infty} e^{-2 x t}\left(\int_{0}^{\infty} e^{-s t} f(s) d s\right)^{p-2} w(t) d t d x \\
& \geq 2 \int_{0}^{\infty} f(x) H f(x) \int_{0}^{\infty} e^{-2 x t}\left(\int_{0}^{x} e^{-x t} f(s) d s\right)^{p-2} w(t) d t d x \\
& =2 \int_{0}^{\infty} f(x) H f(x)^{p-1} \mathscr{L} w(p x) d x .
\end{aligned}
$$

Lemma 2.9. Let $f \geq 0$, let $w$ be a weight, and let $1<p<2$. Then

$$
\int(\mathscr{L} f)^{p} w \geq 2^{p-1} \int f(H f)(x)^{p-1} \mathscr{L} w(p x) d x .
$$

Proof. Here we will use Hölder's inequality twice with exponents $1 /(p-1)$ and $1 /(2-p)$. So

$$
\begin{aligned}
\mathscr{L} w(p x)= & \int_{0}^{\infty} e^{-(p-1) x t}(\mathscr{L} f)^{(p-2)(p-1)}(t)(\mathscr{L} f)^{(2-p)(p-1)}(t) e^{-x t} w(t) d t \\
\leq & {\left[\int_{0}^{\infty} e^{-x t}(\mathscr{L} f)(t)^{p-2} e^{-x t} w(t) d t\right]^{p-1} } \\
& \times\left[\int_{0}^{\infty}(\mathscr{L} f)(t)^{p-1} e^{-x t} w(t) d t\right]^{2-p} \\
= & \mathscr{L} u(2 x)^{p-1} \mathscr{L}\left[w(\mathscr{L} f)^{p-1}\right](x)^{2-p},
\end{aligned}
$$

where $u(x)=w(x)(\mathscr{L} f)(x)^{p-2}$.

Hence,

$$
\begin{aligned}
\int f(H f)(x)^{p-1} \mathscr{L} w(p x) d x & \leq \int f[H f(x) \mathscr{L} u(2 x)]^{p-1} \mathscr{L}\left[w(\mathscr{L} f)^{p-1}\right]^{2-p} \\
& \leq\left[\int f H f(x) \mathscr{L} u(2 x)\right]^{p-1}\left[\int(\mathscr{L} f)^{p} w\right]^{2-p} .
\end{aligned}
$$

But by Lemma 2.8,

$$
\int f H f(x) \mathscr{L} u(2 x) d x \leq \frac{1}{2} \int(\mathscr{L} f)(x)^{2} u(x) d x=\frac{1}{2} \int(\mathscr{L} f)^{p} w,
$$

and the result follows.

Proof of Theorem 2. The various lemmas all show that

$$
\int(\mathscr{L} f)^{q} w \leq C \int f(H f)^{q-1} \mathscr{L} w
$$

and

$$
\int f(H f)^{q-1} \mathscr{L} w(q x) \leq C \int(\mathscr{L} f)^{q} w .
$$

It is clear from these that in order for the Laplace transform $\mathscr{L}: L^{p}(v) \rightarrow$ $L^{q}(w)$, either $\mathscr{L} w<\infty$ a.e., or $v \equiv \infty$ on a set of positive measure; that is 
not allowed, so $\mathscr{L} w$ is finite almost everywhere, and hence $\mathscr{L} w$ decreases to zero as $x \rightarrow \infty$.

It suffices to restrict our attention to a suitable class of test functions, say $f$ continuous, nonnegative, and compactly supported in $(0, \infty)$. Then, integrating by parts, we get

$$
\begin{aligned}
\int f(H f)^{q-1} \mathscr{L} w & =\frac{1}{q-1} \int(H f)^{q}(-d \mathscr{L} w) \\
& =\frac{1}{q-1} \int(H f)(x)^{q} \mathscr{L}[t w(t)](x) d x
\end{aligned}
$$

and so a sufficient condition is that

$$
H: L^{p}(v) \rightarrow L^{q}(-d \mathscr{L} w)
$$

Obviously,

$$
H^{*}(-d \mathscr{L} w)(x)=\mathscr{L} w(x),
$$

so the Hardy conditions in Theorem 2.1 correspond to (1.6) and (1.8).

The same argument with $\int f(H f)^{q-1} \mathscr{L} w(q x) d x$ gives (1.5) and (1.7), and the proof is complete.

\section{Power Weights}

As an application of Theorem 2, we will now describe the necessary and sufficient conditions for power weights for the Laplace transform.

Theorem 3.1. Let $1<p \leq q<\infty$. Then $\mathscr{L}: L^{p}\left(x^{\beta}\right) \rightarrow L^{q}\left(x^{\alpha}\right)$ boundedly if and only if $\alpha>-1$ and $\beta=p-1-p(\alpha+1) / q$.

Proof. With $w(x)=x^{\alpha}$, it follows that $\mathscr{L} w(q x) \approx \mathscr{L} w(x)$, and so the necessary condition (1.5) and the sufficient condition (1.6) of Theorem 2 are equivalent. It is clear that $\mathscr{L} w$ blows up if $\alpha \leq-1$, and so $\alpha>-1$ is needed. In that case $\mathscr{L}\left(x^{\alpha}\right) \approx x^{-\alpha-1}$. We must check the Hardy operator condition (1.6),

$$
H\left(x^{-\beta p^{\prime} / p}\right)^{1 / p^{\prime}} x^{-(\alpha+1) / q} \leq C .
$$

So it is also necessary that $\beta<p-1$, and then this is

$$
x^{1 / p^{\prime}-\beta / p-(\alpha+1) / q} \leq C \quad \text { for all } x,
$$

which forces $\beta=p-1-p(\alpha+1) / q$. Since that $\beta$ is less than $p-1$, this is sufficient as well.

It is clear that condition (1.7) cannot hold for such weights, and so there are no admissible power weights when $q<p$. Weights $v$ of the form $(1+x)^{\beta}$, however, are permissible. Here we obtain

Theorem 3.2. Let $1<q<p<\infty$. For $v(x)$ of the form

$$
v(x)= \begin{cases}1 & \text { if } x \leq 1 \\ x^{\beta} & \text { if } x>1\end{cases}
$$

$\mathscr{L}: L^{p}(v) \rightarrow L^{q}\left(x^{\alpha}\right)$ boundedly if and only if

$$
\max \left\{0,1-\frac{\beta}{p-1}\right\}<\frac{p^{\prime}}{q}(\alpha+1)<1 .
$$


Proof. Again, the necessary condition (1.7) and the sufficient condition (1.8) are equivalent and $\alpha>-1$ is essential. We need to determine when

$$
\int_{0}^{\infty} v^{-p^{\prime} / p}\left[H\left(v^{-p^{\prime} / p}\right)^{1 / q^{\prime}} x^{-(\alpha+1) / q}\right]^{r} d x<\infty,
$$

where $1 / r=1 / q-1 / p$.

Break this integral up from 0 to 1 and from 1 to $\infty$, calling these pieces I and II.

$$
\mathrm{I}=\int_{0}^{\infty}\left[x^{1 / q^{\prime}} x^{-(\alpha+1) / q}\right]^{r} d x
$$

and this is integrable if and only if

$$
r\left[\frac{1}{q^{\prime}}-\frac{\alpha+1}{q}\right]>-1,
$$

which is the condition $p^{\prime}(\alpha+1) / q<1$.

For II, we must distinguish three cases:

Case 1. $\beta>p-1$. Then $H\left(v^{-p^{\prime} / p}\right) \approx 1$.

Case 2. $\beta=p-1$. Then $H\left(v^{-p^{\prime} / p}\right) \approx \log (x+1)$.

Case 3. $\beta<p-1$, where $H\left(v^{-p^{\prime} / p}\right) \approx x^{1-\beta /(p-1)}$.

It is easy to see that II is bounded in Cases 1 and 2. So, when $\beta \leq p-1$, the condition $0<p^{\prime}(\alpha+1) / q<1$ is necessary and sufficient.

That leaves Case 3, where

$$
\mathrm{II} \approx \int_{1}^{\infty} x^{-\beta /(p-1)}\left[x^{(1-\beta /(p-1)) / q^{\prime}-(\alpha+1) / q}\right]^{r} d x,
$$

which is integrable if and only if

$$
\frac{-\beta}{p-1}+r\left[\left(1-\frac{\beta}{p-1}\right) \frac{1}{q^{\prime}}-\frac{\alpha+1}{q}\right]<-1,
$$

which is the condition

$$
1-\frac{\beta}{p-1}<\frac{p^{\prime}}{q}(\alpha+1)
$$

completing the proof of the theorem.

\section{REFERENCES}

1. K. F. Anderson and H. P. Heinig, Weighted norm inequalities for certain integral operators, SIAM J. Math. Anal. 14 (1983), 833-844.

2. M. Artola, untitled and unpublished manuscript.

3. S. Bloom and R. Kerman, Weighted norm inequalities for operators of Hardy type Proc. Amer. Math. Soc. 113 (1991), 135-141.

4. J. S. Bradley, Hardy inequalities with mixed norms, Canad. Math. Bull. 21 (1978), 405-408.

5. W. B. Jurkat and G. Sampson, On maximal rearrangment inequalities for the Fourier transform, Trans. Amer. Math. Soc. 282 (1984), 625-643.

6. V. G. Maz'ja, Sobolev spaces, Springer-Verlag, Heidelberg, 1985.

7. B. Muckenhoupt, Hardy's inequalities with weights, Studia Math. 44 (1972), 31-38. 
8. G. Talenti, Osservazione sopra una Classe di disuguaglianze, Rend. Sem. Mat. Fis. Milano 39 (1969), 171-185.

9. G. Tomaselli, A class of inequalities, Boll. Un. Mat. Ital. 6 (1969), 622-631.

Department of Mathematics, Siena College, Loudonville, New York 12211 